\title{
Association between lipids profile and thyroid parameters in euthyroid diabetic subjects: a cross-sectional study
}

\author{
Yun Zhang ${ }^{1}$, Ping Lu', Ling Zhang ${ }^{1}$ and Xinhua Xiao ${ }^{2^{*}}$
}

\begin{abstract}
Background: The concept is now emerging that higher thyroid-stimulating hormone (TSH) and lower thyroid hormone levels within the euthyroid range may adversely affect atherosclerosis. The aim of this study was to investigate the potential association between thyroid parameters and lipids profile in a cohort of euthyroid diabetic subjects.

Methods: Four hundred and sixty-two euthyroid type 2 diabetes subjects (302 males and 160 females) were consecutively recruited. Clinical and anthropometric data was collected from all participants. Whole blood samples were drawn in the morning after an overnight fasting for the measurement of serum TSH, free thyroxine (FT4), free triiothyronine (FT3), anti-thyroid peroxidase antibody (TPO-Ab) levels, as well as lipid concentrations and glucose.

Results: TSH was higher in females than males. Stratified by TSH, high-density lipoprotein cholesterol (HDL-c) level increased in subjects with TSH $\geq 2.5 \mathrm{ulU} / \mathrm{mL}(P=0.004)$. And TSH was associated with $\mathrm{HDL}-c$ in a Pearson correlation test, however, the association failed to attain significance in partial correlation analyses, adjusted for age, sex, duration of diabetes, fasting glucose and BMI. In females, total cholesterol (TC) and low-density lipoprotein cholesterol (LDL-C) level was significant lower in subjects with TSH $<2.5 \mathrm{ulU} / \mathrm{mL}$. TSH was significantly associated with TC and LDL-c, even in a partial correlation analysis ( $P=0.006$ and 0.011 , respectively). In a multiple linear regression analysis (stepwise), TSH was positive associated with TC $(\beta=0.202, P=0.005)$ and $L D L-C(\beta=0.144, P=0.010)$. In one hundred and six patients having TPO antibody assays, 6 (5.66\%) were positive. The blood pressure and lipid levels were lower in TPO-Ab positive patients, however, the differences were not significantly.

Conclusions: In conclusion, we identified TSH was positively associated with serum TC and LDL-c in euthyroid diabetic women. Our analysis in the subgroup having TPO antibody assays demonstrating non-significantly lower TC levels among seropositive subjects was consistent with the above stated consideration for women as a whole. Further investigations are needed to understand the intimate mechanisms of lipid metabolism in type 2 diabetes with respect to thyroid function.
\end{abstract}

Keywords: Total cholesterol level, Thyroid-stimulating hormone, Euthyroidism, Diabetes, Thyroid antibodies

\section{Background}

Thyroid hormones were recognized as catabolic hormones and they regulated various processes of metabolism, including the synthesis, mobilization, and breakdown of lipids. Hypothyroidism had been reported to be associated with an increased risk for dyslipidemia and atherosclerotic cardiovascular disease $[1,2]$. Interestingly, the concept was

\footnotetext{
* Correspondence: xiaoxinhua@medmail.com.cn

2Department of Endocrinology, Peking Union Medical College Hospital, Chinese Academy of Medical Sciences \& Peking Union Medical College, 100730 Beijing, P R China

Full list of author information is available at the end of the article
}

emerging nowadays that effects of low thyroid function on atherosclerosis susceptibility might extend into the euthyroid range. Several studies reported an association between higher thyroid-stimulating hormone (TSH) and lower thyroid hormone levels that were still within the normal range and lipids profile in the euthyroid population $[3,4]$.

Diabetes mellitus, in particular type 2 diabetes, which was mostly associated with lipoid abnormalities [5], was also known to dramatically increased risk of cardiovascular diseases [6]. Notably, thyroid dysfunctions were more frequent 
in diabetic patients than in the general population [7]. Moreover, the association between circulating TSH levels and cardiovascular diseases risk factors seemed to be amplified by the degree of insulin-resistance [8], and it might be particularly relevant in type 2 diabetes. Thus, glucose, lipids, and thyroid hormones seemed to interact according to a more complex mathematical function than as previously expected. Recently, several studies had reported that even relative low thyroid functions that were still within normal range were more frequent and might be more dangerous in people with diabetes [9-11]. However, only scanty studies on these matters were available. And in most of these studies, free triiodothyronine (FT3), free thyroxine (FT4) and TSH were not measured together.

Taking into account the consideration mentioned above, with the present study, we investigated the potential association between TSH and thyroid hormones levels within the normal range and lipids profile in a cohort of euthyroid type 2 diabetes subjects. We hoped that the information from this study would lead to a better understanding of the relationship between thyroid parameters and lipids profile.

\section{Methods}

\section{Subjects}

Four hundred and sixty-two euthyroid type 2 diabetes subjects (302 males and 160 females) were consecutively recruited in this cross-sectional study from inpatients of Department of Endocrinology, Henan Province People's Hospital. Type 2 diabetes was diagnosed according to American Diabetes Association 2009 criteria [12]. Euthyroidism was defined as TSH, FT3, and FT4 levels within their normal reference ranges (see the next paragraph). Exclusion criteria: type 1 diabetes, latent immune diabetes of the adults, gestational diabetes, and other type of diabetes, pregnancy, neoplasms, as well as any major medical condition in the 6 months preceding the study (i.e. liver, kidney, and heart failure). In particular, subjects with a previous history of thyroid diseases, such as overt hyper/hypothyroidism, thyroid cancer, were excluded. Subjects taking medications affecting thyroid hormone levels (such as thyroid supplementation and antithyroid agents, IFNc, amiodarone, lithium, corticosteroids, etc.) and lipids profile (such as statins, fenofibrate, etc.) were also excluded. Informed consent was obtained from all participants. The present study was approved with the Institutional Ethnics Committee in Zhengzhou University.

\section{Clinical, anthropometric, and laboratory measurements}

Clinical data was collected from all participants. Weight and height were measured with the subjects wearing light clothing and no shoes; the body mass index (BMI) was also calculated $\left(\mathrm{kg} / \mathrm{m}^{2}\right)$. Whole blood samples were drawn in the morning after an overnight fasting for measurements of the study parameters. Serum TSH, FT3, and FT4 levels were measured using chemiluminescence tests (SIEMENS Advia Centaur XP). The normal ranges were as follows: FT3 3.5-6.5pmol/L; FT4 11.5-22.7pmol/L; TSH $0.55-4.78 \mathrm{uIU} / \mathrm{mL}$. Anti-thyroid peroxidase antibody (TPO-Ab) was measured in 106 patients with agreement, with reference values between 0 and 60U/L. The normal range of all these thyroid parameters came from 1,000 normal controls determined before this study. And the inter-assay and intra-assay imprecisions $(\mathrm{CV})$ were all less than 5\%. Fasting plasma glucose (FPG) and lipid concentrations (total cholesterol (TC), triglycerides (TG), high-density lipoprotein cholesterol (HDL-c), and lowdensity lipoprotein cholesterol (LDL-c)) were assayed using enzymatic methods (Roche Diagnostics).

\section{Statistical analyses}

The data analysis was performed using SPSS version18.0. All data was expressed as means \pm standard deviation (SD). All subjects were divided into two groups by sex or TSH $(\geq 2.5 \mathrm{uIU} / \mathrm{mL}$ and $<2.5 \mathrm{uIU} / \mathrm{mL})$. Student's $t$ test or Mann-Whitney $U$ test, depending on the shape of the distribution curves, was used for evaluation of differences between the two groups. The Pearson correlation test was applied in order to assess for the existence of any significant interdependence between numerical parameters. Partial correlation analyses were performed to evaluate the association of thyroid parameters with major cardiovascular risk factors (blood pressure and lipid concentrations), adjusted for age, sex, duration of diabetes, FPG, and BMI. Furthermore, we performed a multiple linear regression analysis (stepwise) to confirm the association, with cardiovascular risk factors as dependent variables, independent variables as follows: age, sex, BMI, duration of diabetes, FPG, FT3, FT4, TSH. Males and females were analyzed separately. Subjects with data of TPO-Ab were divided into two groups: TPO$\mathrm{Ab}$ positive (TPO-Ab $>60 \mathrm{U} / \mathrm{L}$ ) and TPO-Ab negative (TPO-Ab $<60 \mathrm{U} / \mathrm{L}$ ), and differences between the two groups were compared by $t$ tests.

\section{Results}

Table 1 showed the clinical characteristics of this sample of 462 diabetic patients with euthroidsm. The mean age was $54.36 \pm 11.85$ years (range 27 to 84 ), and the mean BMI was $25.33 \pm 3.47 \mathrm{~kg} / \mathrm{m}^{2}$ (range 17.5 to 37.2 ). Age and TSH level were higher in females, and BMI, diastolic blood pressure (DBP), and FT3 were lower. No difference was found in FT4 or duration of diabetes.

Stratified by TSH, HDL-c level increased in subjects with $\mathrm{TSH} \geq 2.5 \mathrm{uIU} / \mathrm{mL}(\mathrm{P}=0.004$, Table 1$)$. And TSH was associated with HDL-c in a Pearson correlation test ( $\beta=0.095, P=0.042$ ), however, the association failed to 
attain significance in partial correlation analyses $(\mathrm{P}=0.092$, Table 2), adjusted for age, sex, duration of diabetes, fasting glucose and BMI.

In females, TC and LDL-c level was significant lower in subjects with TSH $<2.5 \mathrm{uIU} / \mathrm{mL}$ (Table 1 ). TSH was significantly associated with TC and LDL-c, even in a partial correlation analysis $(\mathrm{P}=0.006$ and 0.011 , respectively, Table 2). In a multiple linear regression analysis (stepwise), TSH was positive associated with TC $(\beta=0.202, \mathrm{P}=0.005)$ and LDL-c $(\beta=0.144, P=0.010)$. In males, no difference was found in lipids profile or BP between subjects with $\mathrm{TSH} \geq 2.5 \mathrm{uIU} / \mathrm{mL}$ and $\mathrm{TSH}<2.5 \mathrm{uIU} / \mathrm{mL}$ group (Table 1 ).

In one hundred and six patients with TPO-Ab measured, 6 (5.66\%) were positive and $100(94.34 \%)$ were negative. In TPO-Ab positive patients, SBP, DBP, FT3, TC, TG, HDL, LDL levels were lower than TPO-Ab positive patients. However, the differences were not significantly (Table 3).

\section{Discussion}

Thyroid dysfunction was a risk factor for cardiovascular disease mediated by the effects of thyroid hormones on lipids metabolism and blood pressure [13-15], yet most subjects at risk for cardiovascular disease were euthyroid in the clinical setting. The relationship between thyroid hormones and atherosclerosis in the euthyroid population had garnered much interest recently.

In this study, we demonstrated that TSH was higher in females than males, which was in agreement with previous studies [9]. Furthermore, we found that in diabetic females, TC and LDL-c level was significant lower in subjects with $\mathrm{TSH}<2.5 \mathrm{uIU} / \mathrm{mL}$. TSH was significantly associated with $\mathrm{TC}$ and LDL-c, even in a partial correlation analysis. In a multiple linear regression analysis (stepwise), TSH was positive associated with TC and LDL-c. TSH levels within the reference range had been reported to be associated with serum lipid concentrations previously. Giandalia et al. reported that TSH was associated with visceral obesity and higher triglycerides concentration in type 2 diabetes [9]. And higher TSH was reported to confer increased plasma cholesteryl ester transfer in the context of chronic hyperglycemia in Triolo's study [11]. All these suggested that relatively low but clinically normal thyroid function, as inferred from higher TSH in normal range, could also influence lipids profile and atherosclerosis susceptibility in type 2 diabetes.

One explanation to the positive association of $\mathrm{TSH}$ with TC and LDL-c might be both being a consequence of autoimmune activation involving lipoprotein(a), with ensuing "reduced" lipoprotein(a) levels, a determinant of new-onset diabetes, and accompanied by low circulating TC and LDL-C and autoimmune complex involving $\mathrm{TSH}$ as well. Among euthyroid patients with established diabetes, the stated variables might tend to normalize secondary to a decline in autoimmune processes and in the reduction of lipoprotein(a) levels [16]. And lower TSH in diabetic women associated with lower cholesterol could be attributed to two phenomena: a) Lower TC and LDL-c commonly precede autoimmune-initiated processes -be it oxidatively-damaged TSH or rheumatoid arthritis [17] or diabetes [18]; b) TSH incurring epitope damage escapes immunoassay and is measured as "lower".

Some authors suggested that chronic autoimmune thyroiditis per se might be considered as a risk factor of atherosclerosis independent of thyroid function

Table 1 Clinical characteristics of the subjects

\begin{tabular}{|c|c|c|c|c|c|c|}
\hline & Total & & Males & & Females & \\
\hline$n$ & 462 & & 302 & & 160 & \\
\hline Age(years) & $54.36 \pm 11.85$ & & $53.20 \pm 11.87$ & & $56.56 \pm 11.52$ & \\
\hline Duration of diabetes(years) & $8.33 \pm 6.97$ & & $8.36 \pm 7.10$ & & $8.27 \pm 6.74$ & \\
\hline BMI $\left(\mathrm{kg} / \mathrm{m}^{2}\right)$ & $25.33 \pm 3.47$ & & $25.61 \pm 3.10$ & & $24.80 \pm 4.03$ & \\
\hline FT3(pmol/L) & $4.49 \pm 0.49$ & & $4.62 \pm 0.49$ & & $4.24 \pm 0.38$ & \\
\hline FT4(pmol/L) & $16.60 \pm 2.29$ & & $16.74 \pm 2.27$ & & $16.34 \pm 2.32$ & \\
\hline \multirow[t]{2}{*}{ TSH(ulU/mL) } & $2.05 \pm 0.95$ & & $1.97 \pm 0.89$ & & $2.19 \pm 1.03$ & \\
\hline & $\begin{array}{l}\mathrm{TSH} \geq 2.5 \\
(n=120)\end{array}$ & $\begin{array}{l}\text { TSH }<2.5 \\
(n=342)\end{array}$ & $\begin{array}{l}\mathrm{TSH} \geq 2.5 \\
(\mathrm{n}=69)\end{array}$ & $\begin{array}{l}\text { TSH }<2.5 \\
(n=233)\end{array}$ & $\begin{array}{l}\text { TSH } \geq 2.5 \\
(n=51)\end{array}$ & $\begin{array}{l}\text { TSH }<2.5 \\
(n=109)\end{array}$ \\
\hline $\mathrm{SBP}(\mathrm{mmHg})$ & $133.10 \pm 14.54$ & $133.52 \pm 17.52$ & $132.86 \pm 14.22$ & $133.56 \pm 18.04$ & $133.43 \pm 15.11$ & $133.42 \pm 16.45$ \\
\hline $\mathrm{DBP}(\mathrm{mmHg})$ & $82.51 \pm 8.89$ & $82.41 \pm 9.99$ & $84.14 \pm 8.26$ & $83.42 \pm 10.27$ & $80.29 \pm 9.32$ & $80.24 \pm 9.03$ \\
\hline $\mathrm{TC}(\mathrm{mmol} / \mathrm{L})$ & $4.88 \pm 1.12$ & $4.82 \pm 1.06$ & $4.67 \pm 1.15$ & $4.86 \pm 1.12$ & $5.16 \pm 1.02$ & $4.74 \pm 0.92^{*}$ \\
\hline TG (mmol/L) & $2.04 \pm 2.47$ & $2.11 \pm 1.81$ & $2.35 \pm 3.13$ & $2.29 \pm 2.01$ & $1.62 \pm 0.94$ & $1.72 \pm 1.17$ \\
\hline HDL-c (mmol/L) & $1.18 \pm 0.30$ & $1.09 \pm 0.27^{*}$ & $1.11 \pm 0.28$ & $1.07 \pm 0.27$ & $1.27 \pm 0.31$ & $1.14 \pm 0.26$ \\
\hline LDL-c (mmol/L) & $2.88 \pm 0.84$ & $2.90 \pm 0.83$ & $2.76 \pm 0.85$ & $2.95 \pm 0.88$ & $3.04 \pm 0.81$ & $2.81 \pm 0.71^{*}$ \\
\hline FBG (mmol/L) & $8.55 \pm 3.50$ & $8.97 \pm 3.74$ & $8.51 \pm 3.34$ & $8.96 \pm 3.84$ & $8.60 \pm 3.74$ & $9.00 \pm 3.52$ \\
\hline
\end{tabular}

*P $<0.05$, when compared with TSH $\geq 2.5 \mathrm{umlU} / \mathrm{mL}$. 
Table 2 Correlation of thyroid parameters with lipids and blood pressure in Partial correlation analyses

\begin{tabular}{|c|c|c|c|c|c|c|c|}
\hline & & \multicolumn{2}{|l|}{ FT3 } & \multicolumn{2}{|l|}{ FT4 } & \multicolumn{2}{|l|}{ TSH } \\
\hline & & coefficients & $\mathbf{P}$ & coefficients & $\mathbf{P}$ & coefficients & $P$ \\
\hline \multirow[t]{6}{*}{ Total } & SBP & 0.027 & 0.566 & -0.006 & 0.907 & -0.026 & 0.588 \\
\hline & DBP & 0.035 & 0.453 & -0.009 & 0.845 & 0.017 & 0.719 \\
\hline & TC & 0.012 & 0.794 & 0.010 & 0.835 & 0.044 & 0.352 \\
\hline & TG & 0.034 & 0.472 & 0.004 & 0.933 & -0.003 & 0.948 \\
\hline & $\mathrm{HDL}-\mathrm{C}$ & 0.044 & 0.347 & 0.033 & 0.484 & 0.079 & 0.092 \\
\hline & LDL-C & 0.001 & 0.978 & -0.018 & 0.699 & 0.020 & 0.668 \\
\hline \multirow[t]{6}{*}{ Males } & SBP & 0.028 & 0.630 & -0.043 & 0.466 & -0.046 & 0.430 \\
\hline & DBP & 0.015 & 0.796 & -0.032 & 0.582 & 0.000 & 0.999 \\
\hline & TC & -0.004 & 0.945 & 0.041 & 0.487 & -0.059 & 0.317 \\
\hline & $\mathrm{TG}$ & 0.010 & 0.867 & 0.011 & 0.847 & -0.043 & 0.467 \\
\hline & $\mathrm{HDL}-\mathrm{C}$ & 0.007 & 0.900 & 0.083 & 0.154 & 0.062 & 0.288 \\
\hline & $\mathrm{LDL-C}$ & -0.008 & 0.885 & -0.017 & 0.773 & -0.080 & 0.171 \\
\hline \multirow[t]{6}{*}{ Females } & SBP & 0.022 & 0.787 & 0.064 & 0.433 & 0.001 & 0.992 \\
\hline & DBP & 0.103 & 0.206 & -0.017 & 0.835 & 0.047 & 0.561 \\
\hline & TC & -0.010 & 0.905 & -0.043 & 0.596 & 0.221 & $0.006^{*}$ \\
\hline & $\mathrm{TG}$ & 0.022 & 0.786 & -0.051 & 0.530 & 0.050 & 0.537 \\
\hline & $\mathrm{HDL}-\mathrm{C}$ & 0.147 & 0.069 & -0.064 & 0.431 & 0.113 & 0.163 \\
\hline & LDL-C & -0.038 & 0.642 & 0.002 & 0.982 & 0.204 & $0.011^{*}$ \\
\hline
\end{tabular}

*P $<0.05$.

[19]. In this study, TPO-Ab was considered for the first time in the association of thyroid function and lipids profile and blood pressure in diabetic subjects. Along with higher TSH and lower FT3 values in the seropositive group, lower lipid concentrations and blood pressure were observed, although the differences were not significantly. The result did not support autoimmune thyroiditis as a risk factor for atherosclerosis. This

Table 3 Clinical characteristics of the subjects with data of TPO-Ab

\begin{tabular}{llll}
\hline & TPO-Ab negative & TPO-Ab positive & P \\
\hline N(males/females) & $100(71 / 29)$ & $6(3 / 3)$ & \\
Age $($ years $)$ & $52.71 \pm 11.76$ & $59.67 \pm 10.03$ & 0.160 \\
BMI $\left(\mathrm{kg} / \mathrm{m}^{2}\right)$ & $25.76 \pm 3.38$ & $23.17 \pm 2.21$ & 0.160 \\
$\mathrm{SBP}(\mathrm{mmHg})$ & $133.84 \pm 16.99$ & $126.00 \pm 13.55$ & 0.271 \\
$\mathrm{DBP}(\mathrm{mmHg})$ & $82.50 \pm 9.68$ & $77.83 \pm 8.30$ & 0.251 \\
$\mathrm{FT} 3(\mathrm{pmol} / \mathrm{L})$ & $4.54 \pm 0.45$ & $4.37 \pm 0.45$ & 0.353 \\
$\mathrm{FT} 4(\mathrm{pmol} / \mathrm{L})$ & $16.30 \pm 2.16$ & $16.69 \pm 2.16$ & 0.682 \\
$\mathrm{TSH}(\mathrm{ull} / \mathrm{mL})$ & $2.21 \pm 1.13$ & $2.49 \pm 1.15$ & 0.588 \\
$\mathrm{TC}(\mathrm{mmol} / \mathrm{L})$ & $4.95 \pm 1.15$ & $4.30 \pm 0.48$ & 0.176 \\
$\mathrm{TG}(\mathrm{mmol} / \mathrm{L})$ & $2.06 \pm 2.05$ & $1.59 \pm 1.31$ & 0.578 \\
$\mathrm{HDL}-\mathrm{c}(\mathrm{mmol} / \mathrm{L})$ & $1.17 \pm 0.32$ & $0.95 \pm 0.27$ & 0.099 \\
LDL-c $(\mathrm{mmol} / \mathrm{L})$ & $3.05 \pm 0.94$ & $2.75 \pm 0.40$ & 0.444 \\
FBG $(\mathrm{mmol} / \mathrm{L})$ & $8.40 \pm 3.26$ & $7.74 \pm 5.48$ & 0.647 \\
\hline
\end{tabular}

might result from the small sample size, only 6 patients with TPO-Ab positive. Larger scale studies were needed to further confirm the role of thyroid antibodies in atherosclerosis.

Several methodological aspects and limitations of our study needed to be considered. First of all, the causal relationship could not be inferred from this study because it was cross-sectional in nature. Secondly, in type 2 diabetes patients, the relationship between thyroid hormones and cardiovascular disease risk might be influenced by other diabetes-related variables, such as metabolic control, co-morbidities, and/or hypoglycemic therapies. Strollo et al. found that TC and LDL-C correlated negatively with FT4 and positively with FT3 only in patients treated with insulin, but not in patients treated with oral hypoglycaemic agents [19]. However, treatment of diabetes was not considered in this study.

\section{Conclusion}

In conclusion, we identified TSH was positively associated with serum TC and LDL-c in euthyroid diabetic women. Our analysis in the subgroup having TPO antibody assays demonstrating non-significantly lower TC levels among seropositive subjects was consistent with the above stated consideration for women as a whole. Further investigations are needed to understand the intimate mechanisms of lipid metabolism in type 2 diabetes with respect to thyroid function. 


\section{Abbreviations}

TSH: Thyroid-stimulating hormone; FT3: Free triiodothyronine; FT4: Free thyroxine; BMI: Body mass index; TPO-Ab: Anti-thyroid peroxidase antibody; TC: Total cholesterol; TG: Triglycerides; HDL-c: High-density lipoprotein cholesterol; LDL-c: Low-density lipoprotein cholesterol; SD: Standard deviation; DBP: Diastolic blood pressure; SBP: Systolic blood pressure.

\section{Competing interests}

The authors declare that they have no competing interests.

\section{Authors' contributions}

$Y Z$ and $X X$ wrote the study protocol and designed the study. YZ, PL and $L Z$ collected data and carried out the statistical analysis. $Y Z$ wrote the manuscript. XX contributed to the data interpretation and reviewed and edited the manuscript. All authors read and approved the final manuscript.

\section{Author details}

${ }^{1}$ Department of Endocrinology, Henan Province People's Hospital \& Zhengzhou University People' Hospital, Zhengzhou City, Henan Province, P R China. ${ }^{2}$ Department of Endocrinology, Peking Union Medical College Hospital, Chinese Academy of Medical Sciences \& Peking Union Medical College, 100730 Beijing, P R China.

Received: 7 July 2014 Accepted: 12 March 2015

Published online: 27 March 2015

\section{References}

1. Ichiki T. Thyroid hormone and atherosclerosis. Vascul Pharmacol. 2010:52:151-6.

2. Steinberg AD. Myxedema and coronary artery disease-a comparative autopsy study. Ann Intern Med. 1968;68:338-44.

3. Roos A, Bakker SJ, Links TP, Gans RO, Wolffenbuttel BH. Thyroid function is associated with components of the metabolic syndrome in euthyroid subjects. J Clin Endocrinol Metab. 2007;92:491-6.

4. Garduño-Garcia Jde J, Alvirde-Garcia U, López-Carrasco G, Padilla Mendoza ME, Mehta R, Arellano-Campos O, et al. TSH and free thyroxine concentrations are associated with differing metabolic markers in euthyroid subjects. Eur J Endocrinol. 2010;163:273-8.

5. Cowie CC, Rust KF, Byrd-Holt DD, Gregg EW, Ford ES, Geiss LS, et al. Prevalence of diabetes and high risk for diabetes using $\mathrm{A} 1 \mathrm{C}$ criteria in the U.S. population in 1988-2006. Diabetes Care. 2010;33:562-8.

6. Chamnan P, Simmons RK, Sharp SJ, Griffin SJ, Wareham NJ. Cardiovascular risk assessment scores for people with diabetes: a systematic review. Diabetologia. 2009;52:2001-14.

7. Kadiyala R, Peter R, Okosieme OE. Thyroid dysfunction in patients with diabetes: clinical implications and screening strategies. Int J Clin Pract. 2010;64:1130-9.

8. Chubb SA, Davis WA, Davis TM. Interactions among thyroid function, insulin sensitivity, and serum lipid concentrations: the Fremantle diabetes study. J Clin Endocrinol Metab. 2005:90:5317-20.

9. Giandalia A, Russo GT, Romeo EL, Alibrandi A, Villari P, Mirto AA, et al. Influence of high-normal serum TSH levels on major cardiovascular risk factors and Visceral Adiposity Index in euthyroid type 2 diabetic subjects. Endocrine. 2014:47:152-60.

10. Taneichi H, Sasai T, Ohara M, Honma H, Nagasawa K, Takahashi T, et al. Higher serum free triiodothyronine levels within the normal range are associated with metabolic syndrome components in type 2 diabetic subjects with euthyroidism. Tohoku J Exp Med. 2011;224:173-8.

11. Triolo M, Kwakernaak AJ, Perton FG, de Vries R, Dallinga-Thie GM, Dullaart RP. Low normal thyroid function enhances plasma cholesteryl ester transfer in type 2 diabetes mellitus. Atherosclerosis. 2013;228:466-71.

12. American Diabetes Association. Standards of medical care in diabetes-2009. Diabetes Care. 2009:32 Suppl 1:S13-61.

13. Klein I, Ojamaa K. Thyroid hormone and the cardiovascular system. N Engl J Med. 2001:344:501-9.

14. Cappola AR, Ladenson PW. Hypothyroidism and atherosclerosis. J Clin Endocrinol Metab. 2003:88:2438-44

15. Asvold BO, Vatten LJ, Nilsen TI, Bjøro T. The association between TSH within the reference range and serum lipid concentrations in a population-based study. The HUNT Study. Eur J Endocrinol. 2007;156:181-6.
16. Onat A, Can G, Murat S, Ciçek G, Örnek E, Yüksel H. Aggregation of lipoprotein(a) to apolipoprotein A-I underlying HDL dysfunction as a major coronary risk factor. Anadolu Kardiyol Derg. 2013;13:543-51.

17. Myasoedova E, Crowson CS, Kremers HM, Fitz-Gibbon PD, Therneau TM, Gabriel SE. Total cholesterol and LDL levels decrease before rheumatoid arthritis. Ann Rheum Dis. 2010;69:1310-4.

18. Onat A, Dönmez I, Karadeniz Y, Cakır H, Kaya A. Type-2 diabetes and coronary heart disease: common physiopathology, viewed from autoimmunity. Expert Rev Cardiovasc Ther. 2014;12:667-79.

19. Strollo F, Carucci I, Morè M, Marico G, Strollo G, Masini MA, et al. Free triiodothyronine and cholesterol levels in euthyroid elderly T2DM patients. Int J Endocrinol. 2012;2012:420370.

\section{Submit your next manuscript to BioMed Central and take full advantage of:}

- Convenient online submission

- Thorough peer review

- No space constraints or color figure charges

- Immediate publication on acceptance

- Inclusion in PubMed, CAS, Scopus and Google Scholar

- Research which is freely available for redistribution 